\title{
Article \\ Obesity and Risk of Diabetes Mellitus by Menopausal Status: A Nationwide Cohort Study
}

\author{
Han Rim Lee ${ }^{1}{ }^{(D}$, Jungeun Shin ${ }^{1,2}{ }^{,}$Kyungdo Han ${ }^{3}$, Jiwon Chang ${ }^{1}$, Su-Min Jeong ${ }^{1}$, Seung Joo Chon ${ }^{4}$, \\ Soo Jung Choi ${ }^{5, *}$ and Dong Wook Shin $1,6, * \mathbb{D}$ \\ 1 Department of Family Medicine \& Supportive Care Center, Samsung Medical Center, \\ Sungkyunkwan University School of Medicine, Seoul 06351, Korea; lhl1221@gmail.com (H.R.L.); \\ lovevsv@gmail.com (J.S.); wldnjs5353@gmail.com (J.C.); smjeong.fm@gmail.com (S.-M.J.) \\ 2 International Healthcare Center, Samsung Medical Center, Seoul 06351, Korea \\ 3 Department of Statistics and Actuarial Science, Soongsil University, Seoul 06978, Korea; hkd917@naver.com \\ 4 Department of Obstetrics and Gynecology, Gachon University Gil Medical Center, Incheon 21556, Korea; \\ sjchon@gilhospital.com \\ 5 Department of Family Medicine, Gachon University Gil Medical Center, Incheon 21556, Korea \\ 6 Department of Digital Health, Samsung Advanced Institute for Health Science \& Technology, \\ Sungkyunkwan University, Seoul 06351, Korea \\ * Correspondence: soojchoi3@gilhospital.com (S.J.C.); dwshin.md@gmail.com (D.W.S.)
}

check for updates

Citation: Lee, H.R.; Shin, J.; Han, K.; Chang, J.; Jeong, S.-M.; Chon, S.J.; Choi, S.J.; Shin, D.W. Obesity and Risk of Diabetes Mellitus by Menopausal Status: A Nationwide Cohort Study. J. Clin. Med. 2021, 10, 5189. https://doi.org/10.3390/ jcm10215189

Academic Editors: Andreea Ciudin and Juan M. Pericàs

Received: 4 October 2021

Accepted: 2 November 2021

Published: 6 November 2021

Publisher's Note: MDPI stays neutral with regard to jurisdictional claims in published maps and institutional affiliations.

Copyright: (c) 2021 by the authors. Licensee MDPI, Basel, Switzerland. This article is an open access article distributed under the terms and conditions of the Creative Commons Attribution (CC BY) license (https:/ / creativecommons.org/licenses/by/ $4.0 /)$.

\begin{abstract}
Although both obesity and menopause are associated with increased risk of diabetes mellitus (DM), the association between obesity and DM according to menopausal status remains uncertain. Therefore, we conducted a study to examine the relationship between obesity and incidence of diabetes mellitus (DM) in premenopausal and postmenopausal women. Total of 926,196 premenopausal and 1,193,881 postmenopausal women who underwent health examinations from 2009 to 2014 were identified using the database of the Korean National Health Insurance Service. We compared the incidence and risk of DM according to body mass index (BMI) and waist circumference (WC) in the two groups of women. Cox proportional hazards analyses were performed to evaluate the association between the presence of obesity and risk of DM according to menopausal state. During the 7.8-year follow-up period, 37,736 (4.1\%) premenopausal women and 121,102 (10.1\%) postmenopausal women were diagnosed with DM. Compared to the reference group (BMI 18.5-23), a stronger association between obesity and risk of DM was observed in both pre- and postmenopausal women: multivariable-adjusted hazard ratios and 95\% confidence intervals for BMI subgroups $<18.5,23-25$, $25-30$, and $>30$ were $0.62(0.54,0.70), 1.91(1.85,1.97), 3.38(3.28,3.47)$, and $6.25(6.02,6.48)$, respectively ( $p$ trend $<0.001)$ in premenopausal women and $0.87(0.82,0.92), 1.44(1.41,1.46), 2.00(1.97,2.03)$, and $2.96(2.89,3.02)$ in postmenopausal women ( $p$ trend $<0.001, p$-interaction $<0.001)$. A similar trend was observed for WC. Subgroup analyses of women aged 45 to 55 also showed a stronger association with DM in premenopausal than in postmenopausal women. In conclusion, the association between obesity and DM was stronger in premenopausal women than in postmenopausal women. As estrogens are synthesized in adipose tissue by aromatization of androgens after menopause, increased estrogen levels in obese postmenopausal might have a protective effect against DM.
\end{abstract}

Keywords: diabetes mellitus; obesity; menopause

\section{Introduction}

Diabetes mellitus (DM) is a major public-health problem, with a global prevalence of 9.3\% (463 million people) in 2019 that is expected to reach 10.2\% (578 million people) in 2030 [1]. Obesity, the most prominent risk factor of DM and that leads to the development of insulin resistance, is more common in women than men [2-6]. Women gain about $0.7 \mathrm{~kg} /$ year on average, independent of race [7,8] and more women are overweight or obese after age 45 years, whether more males tend to be overweight at younger age [9]. 
On the other hand, menopause is a potential risk factor for the development of DM, likely due to a reduction in circulating estrogens [10]. The Study of Women's Health Across the Nation (SWAN) suggested that lower E2 concentrations resulted in a 47\% higher risk of type 2 DM during the menopausal transition [11]. The European Prospective Investigation into Cancer (EPIC)-InterAct study suggested that menopause before the age of 40 years was associated with a $32 \%$ greater risk of type $2 \mathrm{DM}$ [12]. A previous meta-analysis reported that levels of fasting glucose (weighted mean difference [WMD] 4.64, 95\% confidence interval [CI] 3.94-5.33) and fasting insulin (WMD 20.88, 95\% CI 2.12-39.65) were both increased after menopause [13].

Randolph et al. reported that obese women had lower pre-menopausal estradiol level, which is sampled on day $2-5$ of a spontaneous menstrual cycle, but higher postmenopausal estradiol level compared to non-obese women [14]. However, the association between obesity and DM according to menopausal status remains unclear. One US study combining the Health Professionals Follow-up Study and the Nurses' Health Study found the association between body mass index (BMI) and risk of DM to be significantly stronger among younger women (age $<60$ years) compared to older women (age 60-69 years and age $\geq 70$ years, $p$ trend $<0.001)$ [15]. While that study included menopausal status as a covariate, the population was generally older (mean age $\sim 64$ years) and thus could not assess the relationship stratified by menopausal status.

Therefore, we conducted a retrospective cohort study to examine the association between obesity and risk of DM in pre- and postmenopausal women using a nationwide population-based data set in Korea.

\section{Materials and Methods}

\subsection{Data Source and Study Setting}

This study used data from the National Health Insurance Service (NHIS), a single government insurer that provides a mandatory universal insurance system that covers approximately $97 \%$ of the Korean population, while the remaining $3 \%$ with low income are covered by the Medical Aid program. Medical service providers submit claims including data on demographics, diagnoses, and medical treatment for reimbursement. In addition, the NHIS provides a biennial National Health Screening Program (NHSP) that includes screening for cardiovascular risk factors, as well as the National Cancer Screening Program (NCSP) that includes breast and cervical cancer screening for all women aged 40 and above [16]. Therefore, the NHIS database contains an eligibility database (age, sex, socioeconomic variables, etc.), a medical treatment database, and a health screening database (health examination results and questionnaires on lifestyle and behavior).

\subsection{Study Population}

Women aged 40 years or older in the NHIS database who had received NHSP and NCSP services from 1 January 2009 to 31 December 2014 were included. Menopausal status was ascertained by self-reported questionnaire. We excluded those with uncertain information on menopausal status $(n=321,984)$, those who were previously diagnosed with DM ( $n=11,470$ in premenopausal, $n=120,228$ in postmenopausal women), and those with high fasting glucose $(\geq 126 \mathrm{mg} / \mathrm{dL})$ at the health examination $(n=30,779$ in premenopausal, $n=134,826$ in postmenopausal women). In addition, those with missing information on reproductive history ( $n=40,334$ in premenopausal, $n=42,911$ in postmenopausal women) and those with other missing covariates ( $n=53,241$ in premenopausal, $n=233,656$ in postmenopausal women) were excluded. Finally, a total of 2,120,077 individuals (926,196 premenopausal and 1,193,881 postmenopausal women) were included in the analysis (Figure 1). 


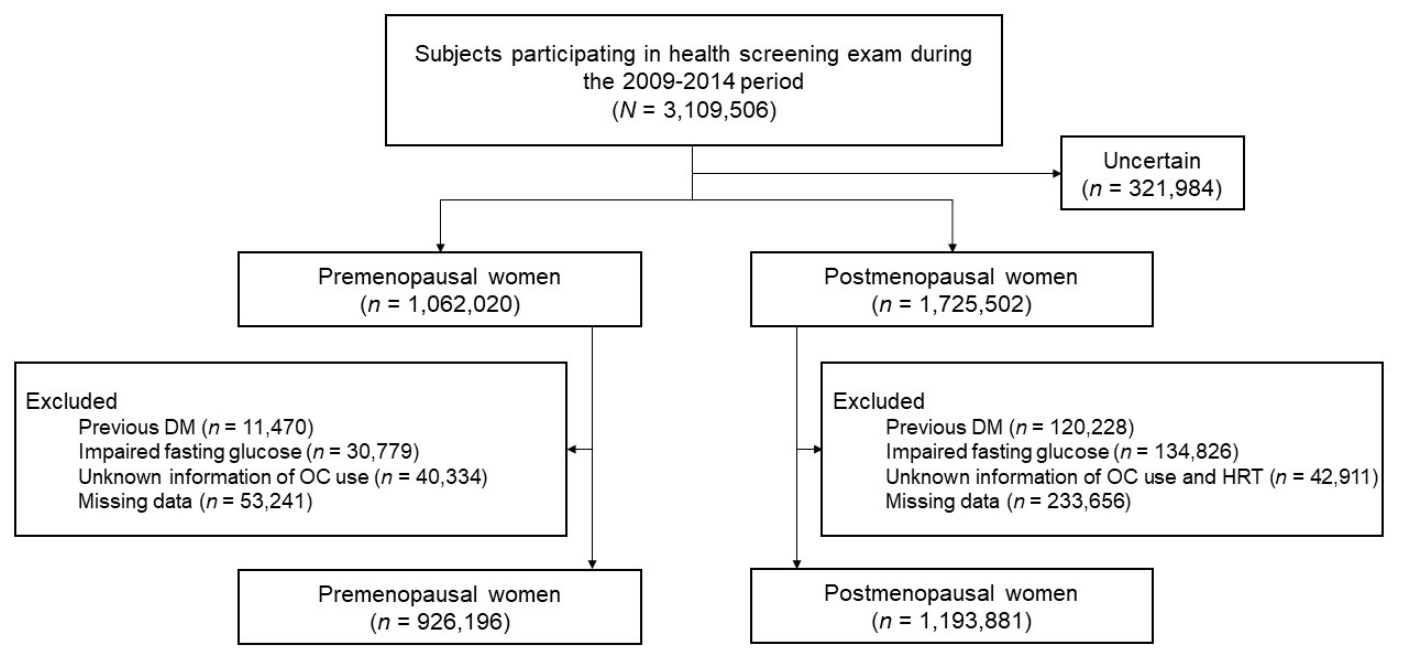

Figure 1. Flow chart of the study population.

\subsection{BMI and WC}

During the health examinations, height and weight were measured. BMI $\left(\mathrm{kg} / \mathrm{m}^{2}\right)$ was calculated using the individual's body weight $(\mathrm{kg})$ divided by the square of height $\left(\mathrm{m}^{2}\right)$ and was categorized as low $(<18.5)$, normal (reference, 18.5-22.9), overweight (23-24.9), obese (25-29.9), or severely obese ( $\geq 30$ ) according to the Asia-Pacific BMI criteria established by the Western Pacific Region of the World Health Organization [17]. Waist circumference (WC) $(\mathrm{cm})$ was measured at the midpoint between the lower margin of the last palpable rib and the top of the iliac crest [18] and was divided into 6 levels: $<75,75-79.9$ (reference category), 80-84.9, 85-89.9, 90-94.9, and $\geq 95 \mathrm{~cm}$.

\subsection{Study Outcomes and Follow-Up}

The primary endpoint of this study was newly diagnosed DM defined by ICD-10 codes for DM (E11-14) with a prescription history of hypoglycemic agents. The cohort was followed from the initial health check-up date to the date of incidence of DM, death, or until the end of the study (31 December 2017), whichever came first.

\subsection{Covariates}

Detailed information on health-related behavior and reproductive history were collected through self-reported questionnaires. Smoking status was categorized into three groups: never, former, and current smokers. Alcohol consumption was assessed as the amount of alcohol consumed per occasion and the frequency of alcohol intake per week and classified into non-, mild ( $<30 \mathrm{~g} /$ day), and heavy drinkers ( $\geq 30 \mathrm{~g} /$ day). Regular physical activity was defined as moderate severity physical activity for more than $30 \mathrm{~min}$ on more than 5 days per week over the past week. The age at menarche was categorized as $\leq 12,13-14,15-16$, and $>16$ years, and the age at menopause was categorized as $<40,40-44$, $45-49,50-54$, or $\geq 55$ years. Participants' reproductive history included parity $(0,1$, or $\geq 2)$, total duration of breastfeeding (never, $<6,6-12$, or $\geq 12$ a total of months), duration of oral contraceptive use (never, $<1$, or $\geq 1$ year), and duration of hormone replacement therapy (HRT) (never, $<2,2-5$, or $\geq 5$ years).

Comorbidities (e.g., hypertension, hyperlipidemia, or chronic kidney disease) were identified based on physician diagnosis or self-reported prescription medication history.

\subsection{Statistical Analysis}

Baseline characteristics were presented as mean \pm standard deviation (SD) for continuous variables and number with proportion for categorical variables. Incidence rates of DM were estimated as the number of events per 1000 person-years. Cox proportional hazards analyses were performed to evaluate the associations between BMI, WC, and risk of DM by 
menopausal state. Model 1 was non adjusted, and Model 2 was adjusted for age, income, smoking, alcohol drinking, regular physical activity, and fasting glucose level. Model 3 was additionally adjusted for comorbidities. Finally, Model 4 was additionally adjusted for reproductive history (parity, duration of breastfeeding, duration of oral contraceptive use, age at menarche, age at menopause, duration of HRT).

As previous studies suggested that age-related factors can affect the association between obesity and DM risk, we stratified the analysis by age (40-49 years, 50-59 years, and $\geq 60$ years) in all participants. As the association between obesity and DM risk could be driven by age rather than hormonal status, we conducted additional analyses in a narrow range of age (45-54 years) known as the perimenopausal transition.

Statistical analyses were performed using SAS version 9.4 (SAS Institute Inc., Cary, NC, USA), and a $p$ value less than 0.05 was considered statistically significant.

\section{Results}

\subsection{Baseline Characteristics of Study Subjects}

Baseline characteristics of the study participants according to menopausal status are described in Table 1. During a mean follow-up of 7.8 years, 37,736 (4.1\%) premenopausal women and 121,102 (10.1\%) postmenopausal women were diagnosed with DM. Participants who were diagnosed with DM were more likely to be older (age 46.5 vs. 44.9 in premenopausal women, 63.1 vs. 61.0 in postmenopausal women) and obese (BMI 25.8 vs. 23.0 in premenopausal women, 25.4 vs. 23.9 in postmenopausal women) in each group according to menopausal status.

Table 1. Baseline characteristics of the study population according to menopausal status.

\begin{tabular}{|c|c|c|c|c|c|c|}
\hline & \multicolumn{3}{|c|}{ Premenopausal } & \multicolumn{3}{|c|}{ Postmenopausal } \\
\hline & \multicolumn{2}{|c|}{ DM } & \multirow{3}{*}{$p$-Value } & \multicolumn{2}{|c|}{ DM } & \multirow{3}{*}{$p$-Value } \\
\hline & $n=\stackrel{\text { No }}{888,460}$ & $\begin{array}{c}\text { Yes } \\
n=37,736\end{array}$ & & $\begin{array}{l}\text { No } \\
n=1,072,779\end{array}$ & $\begin{array}{l}\text { Yes } \\
n=\mathbf{1 2 1}, 102\end{array}$ & \\
\hline & N (\%) & N (\%) & & N (\%) & N (\%) & \\
\hline \multirow{2}{*}{$\begin{array}{l}\text { Age, mean (SD) } \\
\text { Body mass index, } \mathrm{kg} / \mathrm{m}^{2} \text {, } \\
\text { mean (SD) }\end{array}$} & $44.91( \pm 3.9)$ & $46.47( \pm 4.1)$ & $<0.001$ & $61( \pm 8.3)$ & $63.06( \pm 7.9)$ & $<0.001$ \\
\hline & $23.03( \pm 2.9)$ & $25.81( \pm 3.6)$ & $<0.001$ & $23.88( \pm 3.0)$ & $25.44( \pm 3.3)$ & $<0.001$ \\
\hline & $26,271(3.0)$ & $237(0.6)$ & $<0.001$ & $25,901(2.4)$ & $1346(1.1)$ & $<0.001$ \\
\hline $18.5-22.9$ & $455,929(51.3)$ & $7929(21.01)$ & & $400,471(37.3)$ & $24,883(20.6)$ & \\
\hline $23-24.9$ & $207,975(23.4)$ & $8598(22.8)$ & & $288,280(26.9)$ & $29,625(24.5)$ & \\
\hline $25-29.9$ & $177,510(20.0)$ & $16,195(42.9)$ & & $324,374(30.2)$ & $54,754(45.2)$ & \\
\hline \multirow{3}{*}{ Waist circumference, $\mathrm{cm}$} & $20,775(2.3)$ & $4777(12.7)$ & & $33,753(3.2)$ & $10,494(8.7)$ & \\
\hline & $20,170(2.0)$ & (12.1 & $<0.001$ & 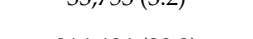 & $10,+7+4(0.1)$ & $<0.001$ \\
\hline & $470,509(53.0)$ & $8121(21.5)$ & & $314,484(29.3)$ & $15,666(12.9)$ & \\
\hline $75-79.9$ & $203,157(22.9)$ & $8472(22.5)$ & & $256,275(23.9)$ & $22,281(18.4)$ & \\
\hline $80-84.9$ & $126,288(14.2)$ & 8838 (23.42) & & $247,831(23.1)$ & $31,458(26.0)$ & \\
\hline $85-89.9$ & $56,024(6.3)$ & $6116(16.21)$ & & $146,726(13.7)$ & $25,296(20.9)$ & \\
\hline 90-94.9 & $21,496(2.4)$ & $3445(9.13)$ & & $71,134(6.6)$ & $15,821(13.1)$ & \\
\hline \multirow{2}{*}{ Smoking status } & $10,986(1.2)$ & $2744(7.27)$ & & $36,329(3.4)$ & $10,580(8.7)$ & \\
\hline & & & $<0.001$ & & & $<0.001$ \\
\hline Never & $844,864(95.1)$ & $35,395(93.8)$ & & $1,034,949(96.5)$ & $115,434(95.3)$ & \\
\hline Ex-smoker & $14,279(1.6)$ & $570(1.5)$ & & $10,902(1.0)$ & $1428(1.2)$ & \\
\hline Current & $29,317(3.3)$ & $1771(4.7)$ & & $26,928(2.5)$ & $4240(3.5)$ & \\
\hline Alcohol drinking & & & $<0.001$ & & & $<0.001$ \\
\hline Non & $635,208(71.5)$ & $28,005(74.2)$ & & $933,632(87.0)$ & $107,606(88.9)$ & \\
\hline Mild & $243,387(27.4)$ & $9125(24.2)$ & & $133,670(12.5)$ & $12,840(10.6)$ & \\
\hline Heavy & $9865(1.1)$ & $606(1.6)$ & & $5477(0.5)$ & $656(0.5)$ & \\
\hline $\begin{array}{l}\text { Regular physical activity } \\
\text { Income }\end{array}$ & $153,878(17.3)$ & $6424(17.0)$ & $\begin{array}{c}0.137 \\
<0.001\end{array}$ & $197,854(18.4)$ & $21,252(17.6)$ & $<0.001$ \\
\hline 1st quartile (lowest) & $227,896(25.7)$ & $10,182(27.0)$ & & $244,674(22.8)$ & $27,725(22.9)$ & \\
\hline 2nd quartile & $179,239(20.2)$ & $8385(22.2)$ & & $199,691(18.6)$ & $22,568(18.6)$ & \\
\hline 3rd quartile & $195,797(22.0)$ & $8556(22.7)$ & & $264,510(24.7)$ & $30,114(24.9)$ & \\
\hline 4th quartile (highest) & $285,528(32.1)$ & $10,613(28.1)$ & & $363,904(33.9)$ & $40,695(33.6)$ & \\
\hline \multicolumn{7}{|l|}{ Comorbid condition } \\
\hline Hypertension & $111,075(12.5)$ & $12,204(32.3)$ & $<0.001$ & $438,377(40.9)$ & $72,790(60.1)$ & $<0.001$ \\
\hline Hyperlipidemia & $86,935(9.8)$ & $8950(23.7)$ & $<0.001$ & $322,240(30.0)$ & $52,569(43.4)$ & $<0.001$ \\
\hline Chronic kidney disease & $36,644(4.1)$ & $1706(4.5)$ & 0.002 & $113,384(10.6)$ & $16,155(13.3)$ & $<0.001$ \\
\hline History of stroke & $2037(0.5)$ & $122(0.6)$ & 0.03 & $12,511(1.8)$ & $2098(2.4)$ & $<0.001$ \\
\hline History of heart disease & $3903(0.9)$ & $348(1.6)$ & $<0.001$ & $34,181(4.9)$ & $6793(7.6)$ & $<0.001$ \\
\hline SBP, $\mathrm{mmHg}$ & $116.7( \pm 14.1)$ & $123.89( \pm 15.6)$ & $<0.001$ & $124.65( \pm 16.0)$ & $129.01( \pm 16.1)$ & $<0.001$ \\
\hline $\mathrm{DBP}, \mathrm{mmHg}$ & $72.85( \pm 9.9)$ & $77.37( \pm 10.5)$ & $<0.001$ & $76.59( \pm 10.1)$ & $78.61( \pm 10.1)$ & $<0.001$ \\
\hline Fasting glucose, $\mathrm{mg} / \mathrm{dL}$ & $91.02( \pm 10.1)$ & $101.4( \pm 12.5)$ & $<0.001$ & $92.94( \pm 10.6)$ & $101.35( \pm 12.4)$ & $<0.001$ \\
\hline Total cholesterol, $\mathrm{mg} / \mathrm{dL}$ & $191.22( \pm 38.3)$ & $204.78( \pm 41.0)$ & $<0.001$ & $208.5( \pm 43.2)$ & $213.16( \pm 45.9)$ & $<0.001$ \\
\hline $\mathrm{HDL}, \mathrm{mg} / \mathrm{dL}$ & $60.72( \pm 35.0)$ & $55.8( \pm 32.1)$ & $<0.001$ & $58.45( \pm 35.7)$ & $55.98( \pm 35.8)$ & $<0.001$ \\
\hline $\mathrm{LDL}, \mathrm{mg} / \mathrm{dL}$ & $114.01( \pm 70.2)$ & $123.07( \pm 60.3)$ & $<0.001$ & $128.07( \pm 68.0)$ & $129.52( \pm 78.3)$ & $<0.001$ \\
\hline $\mathrm{AST}, \mathrm{mg} / \mathrm{dL}$ & $20.33(20.31-20.34)$ & $22.81(22.72-22.9)$ & $<0.001$ & $23.71(23.7-23.72)$ & $25.19(25.14-25.24)$ & $<0.001$ \\
\hline $\mathrm{ALT}, \mathrm{mg} / \mathrm{dL}$ & $16.35(16.33-16.36)$ & $22.08(21.96-22.21)$ & $<0.001$ & $19.44(19.42-19.46)$ & $23.06(22.99-23.12)$ & $<0.001$ \\
\hline $\mathrm{rGTP}, \mathrm{mg} / \mathrm{dL}$ & $16.8(16.78-16.81)$ & $24.36(24.21-24.51)$ & $<0.001$ & $19.62(19.6-19.64)$ & $25.08(25-25.17)$ & $<0.001$ \\
\hline $\mathrm{TG}, \mathrm{mg} / \mathrm{dL}$ & $86.23(86.15-86.32)$ & $125.55(124.87-126.23)$ & $<0.001$ & $111.02(110.91-111.12)$ & $136.95(136.55-137.34)$ & $<0.001$ \\
\hline
\end{tabular}


Table 1. Cont.

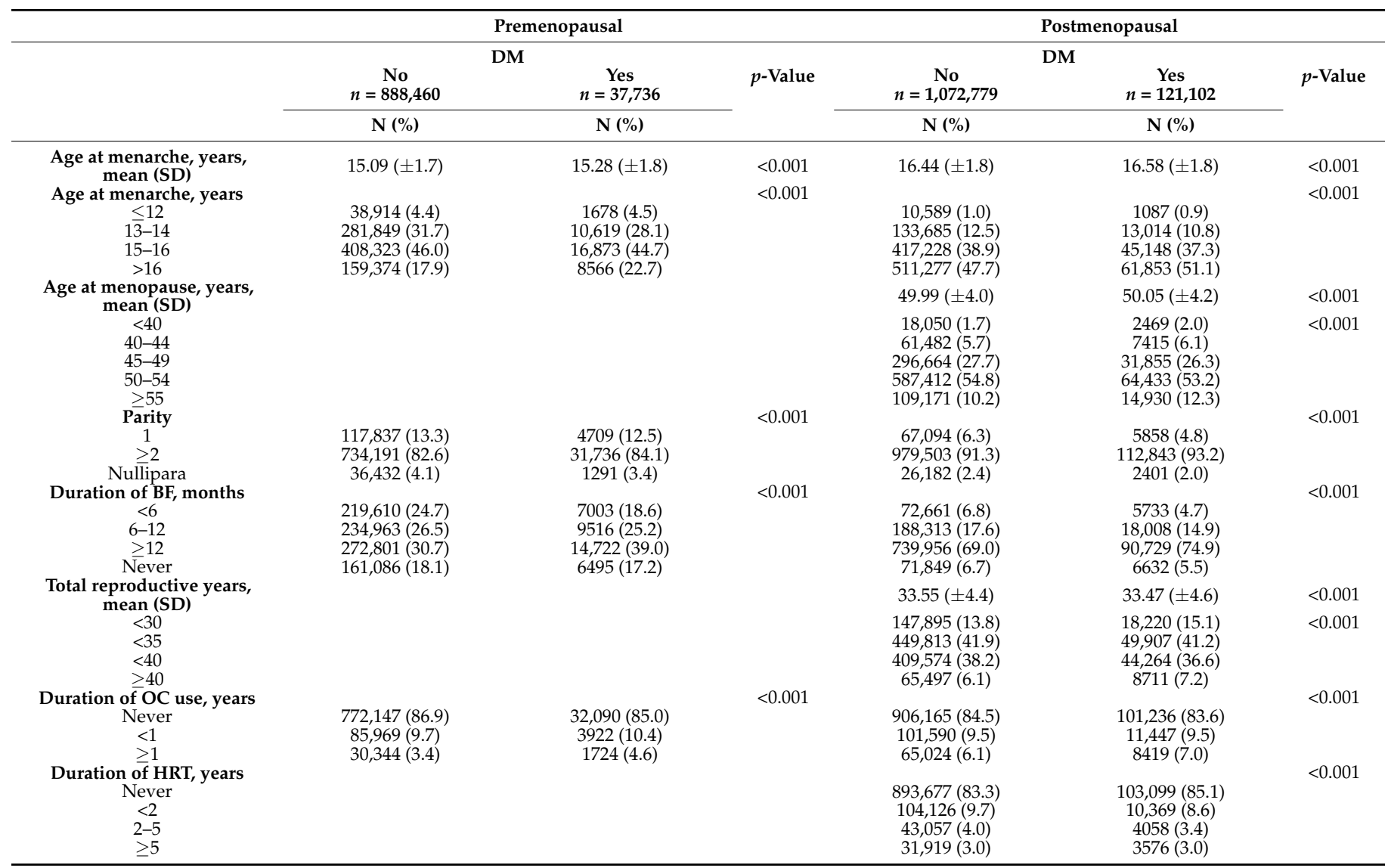

Abbreviations: OC: oral contraceptive, BF: breast feeding, HRT: hormone replacement therapy.

\subsection{Associations of BMI and WC with Risk of DM}

The incidence of DM was 2.07 and 7.28 per 1000 person-years in premenopausal women with normal BMI and postmenopausal women with normal BMI, respectively. Compared with the normal BMI group, adjusted hazard ratio [aHR; $(95 \% \mathrm{CI})$ ] for DM in the $\mathrm{BMI}<18.5,23-25,25-30$, and $>30$ groups was $0.62(0.54,0.70), 1.91(1.85,1.97), 3.38(3.28$, $3.47)$, and $6.25(6.02,6.48)(p$ trend $<0.001)$, respectively, in premenopausal women and 0.87 $(0.82,0.92), 1.44(1.41,1.46), 2.00(1.97,2.00)$, and $2.96(2.89,3.02)$ in postmenopausal women ( $p$ trend $<0.001$; $p$-for-interaction between BMI and menopause $<0.001$ ). A similar trend was observed for WC in both premenopausal and postmenopausal women. However, there was some attenuation of the association between obesity indicators (BMI and WC) and DM risk in postmenopausal women compared with premenopausal women (Table 2 and Figure 2).

\subsection{Analyses Stratified by Age Group}

Table 3 shows the associations of BMI and WC with DM risk stratified by age. Compared with a reference group (BMI 18.5-22.9 $\mathrm{kg} / \mathrm{m}^{2}$, WC $\left.75-79.9 \mathrm{~cm}\right)$, the aHRs $(95 \% \mathrm{CI})$ for DM in the BMI $<18.5,23-25,25-30$, and $>30$ groups were $0.66(0.43,1.02), 1.97$ (1.75, $2.22), 3.17(2.85,3.54)$, and $6.15(5.27,7.17)$, respectively, while those values in the WC $<75,80-84.9,85-89.9,90-94.9$, and $\geq 95$ groups were $0.50(0.45,0.57), 1.37(1.22,1.55), 1.79$ $(1.57,2.04), 2.29(1.95,2.68)$, and $2.91(2.42,3.49)$ in women in their $40 \mathrm{~s}(p$ trend $<0.001)$. In both groups of women aged 50-59 and $\geq 60$ s, aHR increased gradually as BMI and WC increased but not as precipitously as the values of those in their 40s (Figure 3). 
Table 2. Associations between body mass index, waist circumference, and type 2 diabetes risk by menopausal status.

\begin{tabular}{|c|c|c|c|c|c|c|c|c|c|}
\hline & & \multirow{2}{*}{ Subjects $(n)$} & \multirow{2}{*}{ Events $(n)$} & \multirow{2}{*}{$\begin{array}{c}\text { Duration } \\
\text { (Person-Years) }\end{array}$} & \multirow{2}{*}{$\begin{array}{l}\text { Incidence Rate (per } \\
1000 \text { Person-Years) }\end{array}$} & \multicolumn{4}{|c|}{ HR (95\% C.I) } \\
\hline \multicolumn{2}{|c|}{ Body Mass Index } & & & & & Model 1 & Model 2 & Model 3 & Model 4 \\
\hline $\begin{array}{c}\text { Pre- } \\
\text { menopause }\end{array}$ & $\begin{array}{l}<18.5 \\
<23 \\
<25 \\
<30 \\
\geq 30 \\
\quad p\end{array}$ & $\begin{array}{l}\begin{array}{r}26,508 \\
463,858 \\
216,573 \\
193,705 \\
25,552\end{array} \\
\text { lue } \\
\text { rend }\end{array}$ & $\begin{array}{c}237 \\
7929 \\
8598 \\
16,195 \\
4777\end{array}$ & $\begin{array}{c}219,317.7 \\
3,834,458.2 \\
1,775,304.8 \\
1,557,996.3 \\
195,232.5\end{array}$ & $\begin{array}{c}1.08 \\
2.07 \\
4.84 \\
10.40 \\
24.47\end{array}$ & $\begin{array}{c}0.52(0.46,0.60) \\
1 \text { (Ref.) } \\
2.35(2.28,2.42) \\
5.07(4.93,5.20) \\
12.09(11.67,12.53) \\
<0.001 \\
<0.001\end{array}$ & $\begin{array}{c}0.61(0.53,0.69) \\
1 \text { (Ref.) } \\
1.99(1.93,2.05) \\
3.72(3.62,3.82) \\
7.65(7.37,7.93) \\
<0.001 \\
<0.001\end{array}$ & $\begin{array}{c}0.62(0.54,0.70) \\
1 \text { (Ref.) } \\
1.91(1.85,1.97) \\
3.38(3.28,3.47) \\
6.25(6.02,6.48) \\
\quad<0.001 \\
<0.001\end{array}$ & $\begin{array}{c}0.62(0.55,0.71) \\
1 \text { (Ref.) } \\
1.90(1.85,1.96) \\
3.35(3.26,3.45) \\
6.18(5.95,6.41) \\
\quad<0.001 \\
\quad<0.001\end{array}$ \\
\hline $\begin{array}{l}\text { Post- } \\
\text { menopause }\end{array}$ & $\begin{array}{l}<18.5 \\
<23 \\
<25 \\
<30 \\
\geq 30 \\
\quad p\end{array}$ & 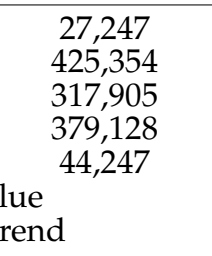 & $\begin{array}{c}1346 \\
24,883 \\
29,625 \\
54,754 \\
10,494\end{array}$ & $\begin{array}{c}211,878.1 \\
3,419,151.6 \\
2,526,788.6 \\
2,933,978.5 \\
323,460.4\end{array}$ & $\begin{array}{c}6.35 \\
7.28 \\
11.72 \\
18.66 \\
32.44\end{array}$ & $\begin{array}{c}0.88(0.83,0.92) \\
1 \text { (Ref.) } \\
1.61(1.59,1.64) \\
2.57(2.53,2.61) \\
4.48(4.38,4.59) \\
<0.001 \\
<0.001\end{array}$ & $\begin{array}{c}0.81(0.77,0.86) \\
1 \text { (Ref.) } \\
1.50(1.48,1.53) \\
2.19(2.16,2.22) \\
3.41(3.34,3.49) \\
\quad<0.001 \\
<0.001\end{array}$ & $\begin{array}{c}0.87(0.82,0.92) \\
1 \text { (Ref.) } \\
1.44(1.41,1.46) \\
2.00(1.97,2.03) \\
2.96(2.89,3.02) \\
<0.001 \\
<0.001\end{array}$ & $\begin{array}{c}0.87(0.83,0.92) \\
1 \text { (Ref.) } \\
1.43(1.41,1.45) \\
1.99(1.96,2.02) \\
2.94(2.87,3.01) \\
<0.001 \\
<0.001\end{array}$ \\
\hline \multicolumn{10}{|c|}{ Waist circumference $(\mathrm{cm})$} \\
\hline $\begin{array}{c}\text { Pre- } \\
\text { menopause }\end{array}$ & $\begin{array}{l}<75 \\
<80 \\
<85 \\
<90 \\
<95 \\
\geq 95 \\
\quad \quad p\end{array}$ & $\begin{array}{l}\begin{array}{r}478,630 \\
211,629 \\
135,126 \\
62,140 \\
24,941 \\
13,730\end{array} \\
\text { lue } \\
\text { rend }\end{array}$ & $\begin{array}{l}8121 \\
8472 \\
8838 \\
6116 \\
3445 \\
2744\end{array}$ & $\begin{array}{c}3,955,873.2 \\
1,734,159.5 \\
1,095,861.7 \\
496,785.1 \\
195,616.5 \\
104,013.5\end{array}$ & \begin{tabular}{|l|}
2.05 \\
4.89 \\
8.07 \\
12.31 \\
17.61 \\
26.38
\end{tabular} & $\begin{array}{c}0.42 \text { (0.41, } 0.43) \\
1 \text { (Ref.) } \\
1.66(1.61,1.71) \\
2.54(2.45,2.62) \\
3.65(3.50,3.79) \\
5.51 \text { (5.28, } 5.75) \\
<0.001 \\
<0.001\end{array}$ & $\begin{array}{c}0.50(0.48,0.51) \\
1 \text { (Ref.) } \\
1.49(1.44,1.53) \\
2.09(2.02,2.16) \\
2.80(2.69,2.92) \\
3.90(3.73,4.07) \\
\quad<0.001 \\
<0.001\end{array}$ & $\begin{array}{c}0.52 \text { (0.50, } 0.53) \\
1 \text { (Ref.) } \\
1.44(1.39,1.48) \\
1.93(1.87,2.00) \\
2.49(2.39,2.59) \\
3.27(3.13,3.42) \\
\quad<0.001 \\
<0.001\end{array}$ & $\begin{array}{c}0.52 \text { (0.51, 0.54) } \\
1 \text { (Ref.) } \\
1.43(1.39,1.47) \\
1.92(1.86,1.98) \\
2.47(2.37,2.57) \\
3.24(3.10,3.38) \\
\quad<0.001 \\
<0.001\end{array}$ \\
\hline $\begin{array}{l}\text { Post- } \\
\text { menopause }\end{array}$ & $\begin{array}{l}<75 \\
<80 \\
<85 \\
<90 \\
<95 \\
\geq 95 \\
\quad \quad p\end{array}$ & $\begin{array}{l}330,150 \\
278,556 \\
279,289 \\
172,022 \\
86,955 \\
46,909 \\
\text { lue } \\
\text { rend }\end{array}$ & $\begin{array}{l}15,666 \\
22,281 \\
31,458 \\
25,296 \\
15,821 \\
10,580\end{array}$ & $\begin{array}{c}2,673,318.7 \\
2,226,416.4 \\
2,195,118.2 \\
1,323,517.1 \\
65,4781.3 \\
342,105.5\end{array}$ & \begin{tabular}{|l|}
5.86 \\
10.01 \\
14.33 \\
19.11 \\
24.16 \\
30.93
\end{tabular} & $\begin{array}{c}0.59 \text { (0.57, } 0.60) \\
1 \text { (Ref.) } \\
1.43(1.41,1.46) \\
1.91(1.88,1.95) \\
2.42(2.38,2.47) \\
3.11(3.04,3.18) \\
<0.001 \\
<0.001\end{array}$ & $\begin{array}{c}0.64(0.63,0.66) \\
1 \text { (Ref.) } \\
1.33(1.31,1.36) \\
1.67(1.64,1.70) \\
1.99(1.94,2.03) \\
2.38(2.32,2.44) \\
<0.001 \\
<0.001\end{array}$ & $\begin{array}{c}0.67(0.66,0.69) \\
1 \text { (Ref.) } \\
1.29(1.27,1.31) \\
1.58(1.55,1.61) \\
1.84(1.80,1.88) \\
2.15(2.10,2.20) \\
<0.001 \\
<0.001\end{array}$ & $\begin{array}{c}0.67(0.66,0.69) \\
1 \text { (Ref.) } \\
1.29(1.27,1.31) \\
1.58(1.55,1.61) \\
1.84(1.80,1.88) \\
2.15(2.10,2.20) \\
\quad<0.001 \\
<0.001\end{array}$ \\
\hline
\end{tabular}

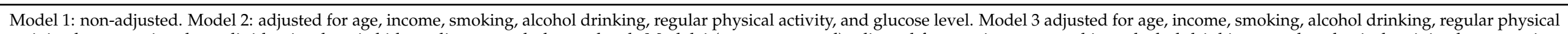

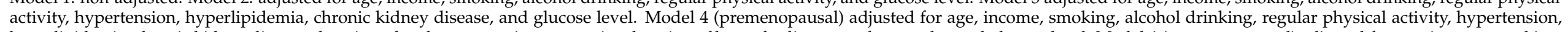

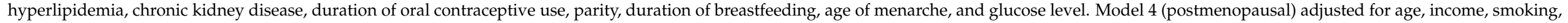

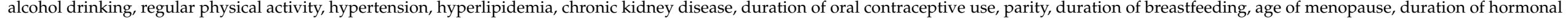
replacement therapy, and glucose level. 

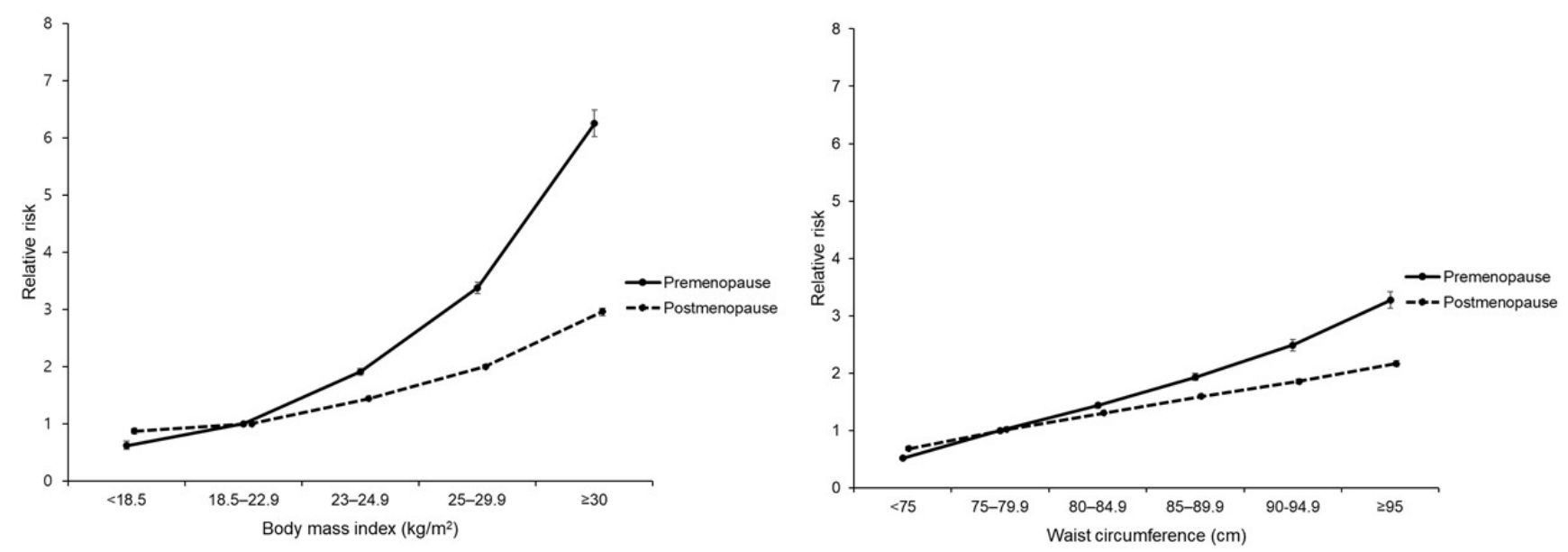

Figure 2. Diabetes incidence according to body mass index and waist circumference by menopausal status.
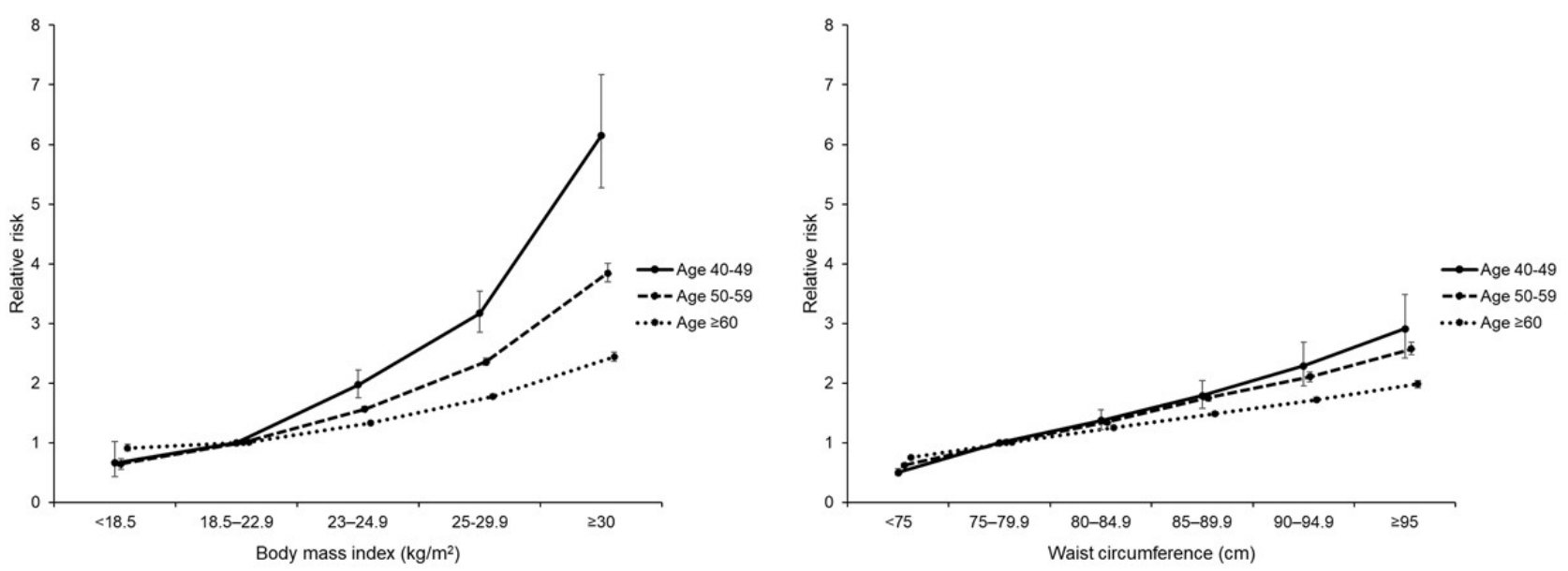

Figure 3. Diabetes incidence according to body mass index and waist circumference by age group.

\subsection{Analyses with Transitional Age}

Among participants aged 45-54 years, aHR values for DM in the BMI $<18.5,23-25$, $25-30$, and $>30$ groups were $0.62(0.52,0.75), 1.76(1.69,1.83), 3.04(2.93,3.14)$, and $5.44(5.18$, $5.72)$ in premenopausal women and $0.60(0.48,0.74), 1.74(1.66,1.83), 2.69(2.57,2.81)$, and $4.57(4.27,4.90)$ in postmenopausal women, respectively ( $p$ trend $<0.001 ; p$-for-interaction between BMI and menopause $<0.001$ ). A similar trend was observed for WC in both premenopausal and postmenopausal women $(p$ trend $<0.001$; $p$-for-interaction between WC and menopause $<0.001$ ) (Table 4 and Figure 4 ). 
Table 3. Associations between body mass index, waist circumference, and type 2 diabetes risk by age group.

\begin{tabular}{|c|c|c|c|c|c|c|c|c|}
\hline \multirow{2}{*}{\multicolumn{2}{|c|}{ Body Mass Index }} & \multirow{2}{*}{ Subjects $(n)$} & \multirow{2}{*}{ Events $(n)$} & \multirow{2}{*}{$\begin{array}{c}\text { Duration } \\
\text { (Person-Years) }\end{array}$} & \multirow{2}{*}{$\begin{array}{c}\text { Incidence Rate (per } 1000 \\
\text { Person-Years) }\end{array}$} & \multicolumn{3}{|c|}{ HR (95\% C.I) } \\
\hline & & & & & & Model 1 & Model 2 & Model 3 \\
\hline Age $40-49$ & $\begin{array}{c}<18.5 \\
<23 \\
<25 \\
<30 \\
\geq 30 \\
p \text {-value } \\
p \text { for trend }\end{array}$ & $\begin{array}{c}25,864 \\
426,550 \\
188,091 \\
163,509 \\
21,806\end{array}$ & $\begin{array}{c}228 \\
6640 \\
6864 \\
12,653 \\
3899\end{array}$ & $\begin{array}{c}213,778.1 \\
3,526,027.9 \\
1,542,779.7 \\
1,317,875.7 \\
167,153.0\end{array}$ & $\begin{array}{c}1.07 \\
1.88 \\
4.45 \\
9.60 \\
23.33\end{array}$ & $\begin{array}{c}0.57 \text { (0.50, } 0.65) \\
1 \text { (Ref.) } \\
2.37 \text { (2.29, } 2.45) \\
5.14(4.99,5.29) \\
12.67(12.17,13.18) \\
<0.001 \\
<0.001\end{array}$ & $\begin{array}{c}0.64 \text { ( }(0.42,0.98) \\
1 \text { (Ref.) } \\
2.09(1.86,2.36) \\
3.54(3.18,3.94) \\
7.61(6.54,8.84) \\
<0.001 \\
<0.001\end{array}$ & $\begin{array}{l}0.66(0.43,1.02) \\
1 \text { (Ref.) } \\
1.97(1.75,2.22) \\
3.17(2.85,3.54) \\
6.15(5.27,7.17) \\
\quad<0.001 \\
<0.001\end{array}$ \\
\hline Age 50-59 & $\begin{array}{c}<18.5 \\
<23 \\
<25 \\
<30 \\
\geq 30 \\
p \text {-value } \\
p \text { for trend }\end{array}$ & $\begin{array}{c}10,953 \\
254,769 \\
176,462 \\
184,147 \\
21,554\end{array}$ & $\begin{array}{c}233 \\
9720 \\
12,209 \\
22,235 \\
5025\end{array}$ & $\begin{array}{c}90,262.1 \\
2,093,159.6 \\
1,431,781.9 \\
1,457,318.4 \\
160,396.5\end{array}$ & $\begin{array}{c}2.58 \\
4.64 \\
8.53 \\
15.26 \\
31.33\end{array}$ & $\begin{array}{c}0.56(0.49,0.63) \\
1 \text { (Ref.) } \\
1.84(1.79,1.89) \\
3.30(3.22,3.38) \\
6.85(6.61,7.08) \\
<0.001 \\
<0.001\end{array}$ & $\begin{array}{c}0.60 \text { ( }(0.52,0.69) \\
1 \text { (Ref.) } \\
1.64(1.59,1.69) \\
2.59(2.52,2.66) \\
4.57(4.40,4.75) \\
\quad<0.001 \\
<0.001\end{array}$ & $\begin{array}{l}0.64 \text { ( }(0.55,0.73) \\
1 \text { (Ref.) } \\
1.56(1.52,1.61) \\
2.35(2.29,2.42) \\
3.84(3.69,4.00) \\
<0.001 \\
<0.001\end{array}$ \\
\hline Age $\geq 60$ & $\begin{array}{c}<18.5 \\
<23 \\
<25 \\
<30 \\
\geq 30 \\
p \text {-value } \\
p \text { for trend } \\
<\text { (cm) }\end{array}$ & $\begin{array}{c}16,938 \\
207,893 \\
169,925 \\
225,177 \\
26,439\end{array}$ & $\begin{array}{c}1122 \\
16,452 \\
19,150 \\
36,061 \\
6347\end{array}$ & $\begin{array}{c}127,155.6 \\
1,634,422.3 \\
1,327,531.8 \\
1,716,780.6 \\
191,143.4\end{array}$ & $\begin{array}{l}8.82 \\
10.07 \\
14.43 \\
21.01 \\
33.21\end{array}$ & $\begin{array}{c}0.88(0.82,0.93) \\
1 \text { (Ref.) } \\
1.43(1.40,1.46) \\
2.09(2.05,2.13) \\
3.31(3.21,3.40) \\
<0.001 \\
<0.001\end{array}$ & $\begin{array}{c}0.86 \text { ( }(0.81,0.91) \\
1 \text { (Ref.) } \\
1.39(1.36,1.42) \\
1.91(1.87,1.94) \\
2.75(2.67,2.83) \\
<0.001 \\
<0.001\end{array}$ & $\begin{array}{l}0.91 \text { ( } 0.86,0.97) \\
1 \text { (Ref.) } \\
1.33(1.30,1.36) \\
1.77(1.74,1.80) \\
2.44(2.36,2.51) \\
<0.001 \\
<0.001\end{array}$ \\
\hline Age $40-49$ & $\begin{array}{c}<75 \\
<80 \\
<85 \\
<90 \\
<95 \\
\geq 95 \\
p \text {-value } \\
p \text { for trend }\end{array}$ & $\begin{array}{l}441,948 \\
184,815 \\
114,722 \\
52,003 \\
20,804 \\
11,528\end{array}$ & $\begin{array}{l}6887 \\
6849 \\
6878 \\
4792 \\
2679 \\
2199\end{array}$ & $\begin{array}{c}3,652,464.9 \\
1,515,174.3 \\
931,954.0 \\
416,527.5 \\
163,718.1 \\
87,775.5\end{array}$ & $\begin{array}{l}1.89 \\
4.52 \\
7.38 \\
11.51 \\
16.36 \\
25.05\end{array}$ & $\begin{array}{c}0.42 \text { (0.40, } 0.43) \\
1 \text { (Ref.) } \\
1.64 \text { (1.58, } 1.69) \\
2.56(2.47,2.66) \\
3.66(3.50,3.83) \\
5.66(5.39,5.94) \\
<0.001 \\
<0.001\end{array}$ & $\begin{array}{c}0.48 \text { ( } 0.42,0.54) \\
1 \text { (Ref.) } \\
1.42 \text { (1.26, } 1.60) \\
1.93(1.69,2.20) \\
2.57(2.20,3.01) \\
3.48(2.90,4.17) \\
<0.001 \\
<0.001\end{array}$ & $\begin{array}{l}0.50(0.45,0.57) \\
1 \text { (Ref.) } \\
1.37(1.22,1.55) \\
1.79(1.57,2.04) \\
2.29(1.95,2.68) \\
2.91(2.42,3.49) \\
<0.001 \\
<0.001\end{array}$ \\
\hline Age 50-59 & $\begin{array}{c}<75 \\
<80 \\
<85 \\
<90 \\
<95 \\
\geq 95 \\
p \text {-value } \\
p \text { for trend }\end{array}$ & $\begin{array}{l}230,613 \\
164,648 \\
134,782 \\
70,762 \\
31,062 \\
16,018\end{array}$ & $\begin{array}{c}7814 \\
10,467 \\
12,715 \\
9377 \\
5355 \\
3694\end{array}$ & $\begin{array}{c}1,898,605.6 \\
1,338,453.7 \\
1,080,880.5 \\
556,687.1 \\
239,324.6 \\
118,967.1\end{array}$ & $\begin{array}{l}4.12 \\
7.82 \\
11.76 \\
16.84 \\
22.38 \\
31.05\end{array}$ & $\begin{array}{c}0.53 \text { (0.51, } 0.54) \\
1 \text { (Ref.) } \\
1.51 \text { (1.47, } 1.55) \\
2.16(2.10,2.23) \\
2.88(2.79,2.98) \\
4.02(3.87,4.17) \\
<0.001 \\
<0.001\end{array}$ & $\begin{array}{c}0.59 \text { (0.58, } 0.61) \\
1 \text { (Ref.) } \\
1.38 \text { (1.34, } 1.42) \\
1.86(1.80,1.92) \\
2.29(2.21,2.38) \\
2.93(2.81,3.05) \\
<0.001 \\
<0.001\end{array}$ & $\begin{array}{l}0.62(0.60,0.64) \\
1 \text { (Ref.) } \\
1.34(1.30,1.38) \\
1.75(1.69,1.80) \\
2.10(2.02,2.18) \\
2.57(2.47,2.68) \\
<0.001 \\
<0.001\end{array}$ \\
\hline Age $\geq 60$ & $\begin{array}{c}<75 \\
<80 \\
<85 \\
<90 \\
<95 \\
<95 \\
p \text {-value } \\
p \text { for trend }\end{array}$ & $\begin{array}{c}136,219 \\
140,722 \\
164,911 \\
111,397 \\
60,030 \\
33,093\end{array}$ & $\begin{array}{c}9086 \\
13,437 \\
20,703 \\
17,243 \\
11,232 \\
7431\end{array}$ & $\begin{array}{c}1,078,121.3 \\
1,106,947.8 \\
1,278,145.4 \\
847,087.6 \\
447,355.1 \\
239,376.5\end{array}$ & $\begin{array}{l}8.43 \\
12.14 \\
16.20 \\
20.36 \\
25.11 \\
31.04\end{array}$ & $\begin{array}{c}0.69 \text { ( }(0.68,0.71) \\
1 \text { (Ref.) } \\
1.34 \text { (1.31, } 1.36) \\
1.68(1.64,1.72) \\
2.07(2.02,2.12) \\
2.56(2.49,2.64) \\
<0.001 \\
<0.001\end{array}$ & $\begin{array}{c}0.72 \text { (0.70, } 0.74) \\
1 \text { (Ref.) } \\
1.28 \text { (1.26, } 1.31) \\
1.55(1.52,1.59) \\
1.83(1.79,1.88) \\
2.15(2.09,2.21) \\
<0.001 \\
<0.001\end{array}$ & $\begin{array}{c}0.75(0.73,0.77) \\
1 \text { (Ref.) } \\
1.25(1.22,1.28) \\
1.48(1.45,1.52) \\
1.72(1.68,1.76) \\
1.98(1.92,2.04) \\
\quad<0.001 \\
<0.001\end{array}$ \\
\hline
\end{tabular}

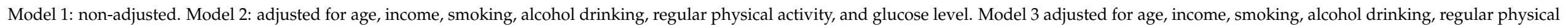
activity, hypertension, hyperlipidemia, chronic kidney disease, and glucose level. 
Table 4. Associations between body mass index, waist circumference, and type 2 diabetes risk by menopausal status in 45-54-year-old women.

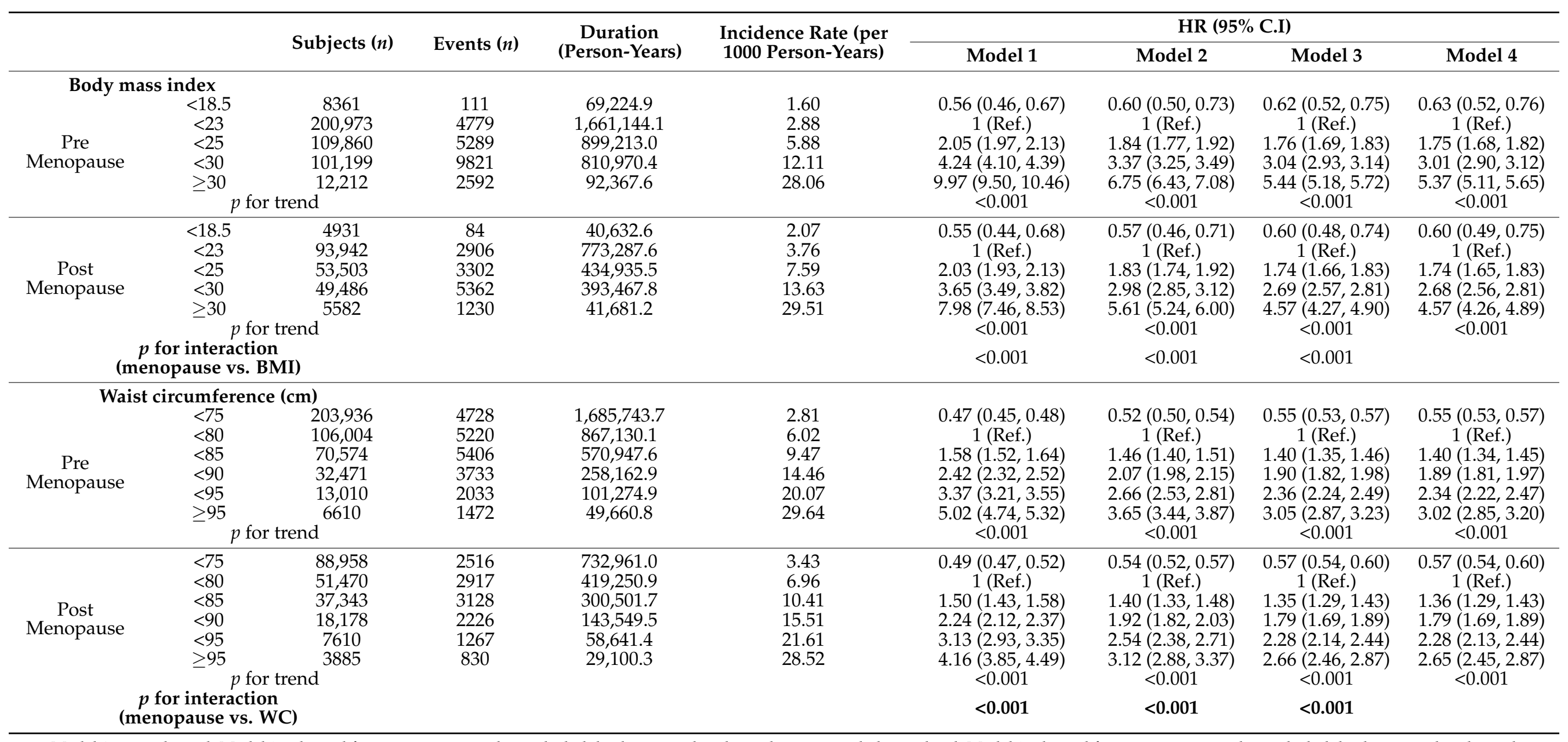

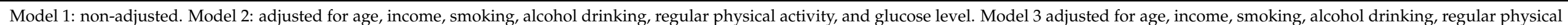

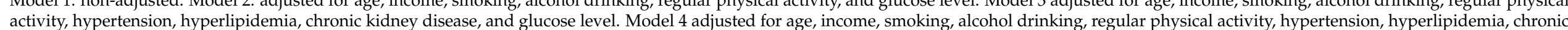

kidney disease, duration of oral contraceptive use, parity, duration of breastfeeding, age of menopause, duration of hormonal replacement therapy, and glucose level. 

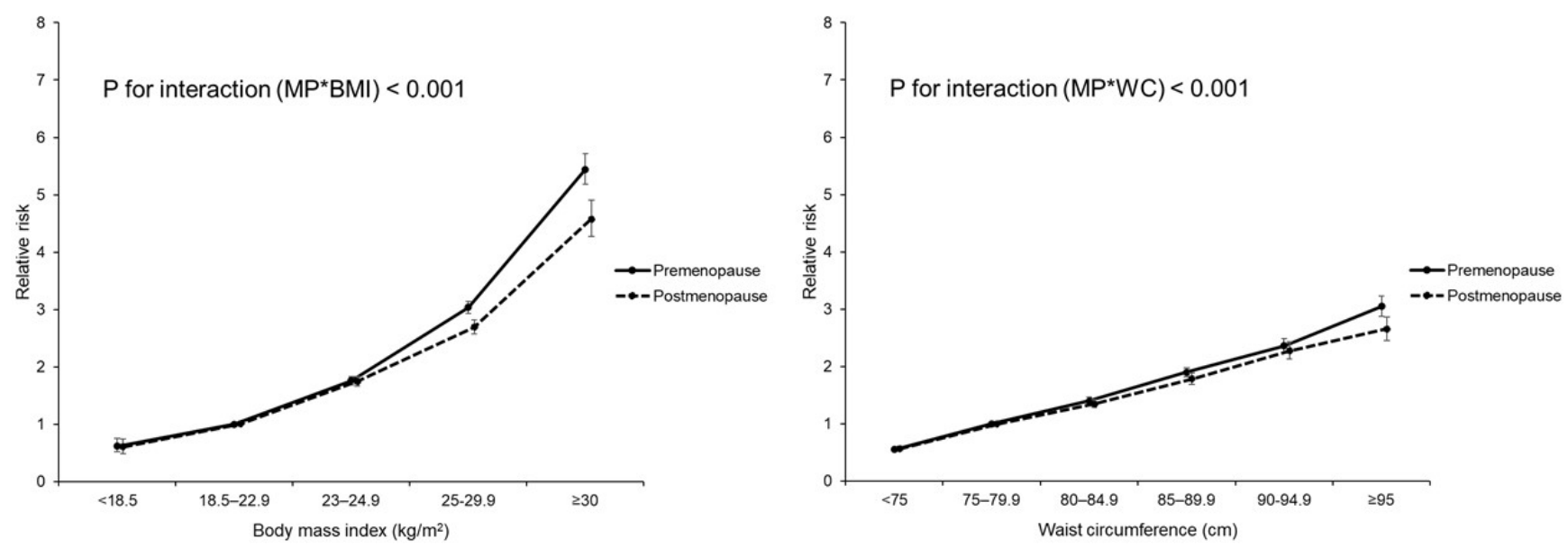

Figure 4. Diabetes incidence according to body mass index and waist circumference by menopausal status in 45-55-yearold women.

\section{Discussion}

In this study, we investigated the association between obesity as defined by indicators (BMI and WC) and risk of DM in premenopausal and postmenopausal women. We found that obesity was associated with increased risk of DM in both groups but with a stronger association in premenopausal women than in postmenopausal women. In sensitivity analysis with transitional aged women (45-54 years) considering an aging effect on DM incidence, a consistently stronger association was found in premenopausal women than postmenopausal women.

The increased risk of developing DM after menopause is due to a decrease in estrogen. Weight gain tends to accompany aging in a woman's life cycle and has been suggested to be a result of decreasing estrogen level after menopause, as estrogen facilitates adipose tissue function and deposition [19]. Thus, menopause is followed by adipose tissue redistribution to visceral depots, which is associated with insulin resistance, while greater subcutaneous gluteal-femoral fat is associated with protection from metabolic syndrome [20].

On the other hand, the impact of body weight on DM risk differs in obese women depending on menopausal status. In premenopausal women, obesity has a direct inhibitory effect on estradiol production from the ovaries [21,22] while estrogens are synthesized in adipose tissue by aromatization of androgens after menopause [23]. Indeed, estradiol level is lower in obese women than non-obese women at premenopausal age but is higher in obese women of post-menopausal age [14]. The present study shows that the association between obesity and DM was more prominent in premenopausal women than in postmenopausal women. It seems that obesity before menopause increases the risk of DM by lowering the estrogen level in the body in addition to an increase in insulin resistance caused by obesity itself. On the contrary, obesity after menopause can increase the estrogen level in the body, providing a protective effect against DM.

The different tendencies of association between obesity and DM by menopausal status can be explained by the change of body composition during the menopause transition. During pre-menopause, fat mass tends to increase, while the proportion of lean mass decreases over time. However, in Chinese women, decreasing fat mass and increasing proportional lean mass were found after menopausal transition [24]. Therefore, high BMI might reflect excess fat mass, which contributes to the association between obesity and $\mathrm{DM}$, in premenopausal women rather than postmenopausal women.

Our study results might be confounded as menopause is linked with aging itself. Therefore, we limited our analyses to women aged $45-54$ years, when menopause generally occurs. Even after adjusting for age and other confounding factors, a stronger association 
was found in premenopausal women, supporting the main results of our study and the above explanations.

Clinical implications of our study are as follows. First, it is important to reduce obesity in premenopausal women. A previous study investigated the age differences between BMI and DM incidence and showed relatively higher risk in younger age [aHR $(95 \% \mathrm{CI}) 4.72$ $(1.79,12.40)$ in 30-39 aged women, $1.54(1.02,2.33)$ in 50-59 aged women] [25]. The Asia Pacific Cohort Studies Collaboration also suggested stronger relationships between BMI and risk of DM in younger women (for each reduction in BMI of $2 \mathrm{~kg} / \mathrm{m}^{2}, 31 \%$ lower risk in group age $\leq 60,19 \%$ lower risk in group age $>70$, and $27 \%$ lower risk in women compared to $23 \%$ lower risk in men) [26]. As diabetic women manifest heightened cardiovascular mortality compared to diabetic men, it is important to prevent DM in women [20,27]. Second, given that estrogen might have a protective effect, HRT might be helpful to prevent diabetes mellitus, especially in lean women. In the large Nurses' Health Study, current users of hormonal therapy had reduced incidence of DM; relative risk $0.80(0.67,0.96)$ compared to non-users after adjusting for age and BMI [28]. The WHI and HERS trials also showed similar findings $[29,30]$. Our results provide additional evidence for the potential benefit of HRT in the prevention of DM and cardiovascular disease, although use of HRT should be based on the balance of benefits and risks.

This study has several limitations. First, we did not directly measure the fat mass, which was more strongly associated with the risk of DM than was BMI, although BMI typically is a good indicator of body fatness [31]. Second, as this study predominantly included people who underwent health examinations, participants tended to be healthier than the general population. Third, we obtained information about menopause based on a self-administered questionnaire, which might have caused misclassification. Fourth, as the study design was observational, it was not possible to distinguish the effects of age from those of menopause. Lastly, although we adjusted for known confounders, we cannot rule out unmeasured and residual confounding. Despite these limitations, this study included a large representative sample and used both BMI and WC indicators of obesity. To our knowledge, this is the first large cohort study to evaluate the association between obesity and risk of DM incidence separately in pre- and postmenopausal women.

In conclusion, this study found a stronger association between obesity and risk of DM among premenopausal women compared with postmenopausal women in the Korean population. Future studies are needed to better understand the precise mechanism of the different relationships between obesity and incidence of diabetes by menopausal status.

Author Contributions: Study conception and design, H.R.L., S.J.C. (Soo Jung Choi) and D.W.S.; data acquisition and analysis, K.H. and D.W.S.; data interpretation and manuscript writing, H.R.L., D.W.S. and S.J.C. (Soo Jung Choi); review and editing, J.S., J.C., S.-M.J., S.J.C. (Seung Joo Chon) and D.W.S. All authors have read and agreed to the published version of the manuscript.

Funding: This research received no external funding.

Institutional Review Board Statement: The study protocol was approved by the Institutional Review Board (IRB) of Samsung Medical Center (IRB No. 2019-07-045).

Informed Consent Statement: The need for informed consent was waived.

Data Availability Statement: The data presented in this study could be accessed via the Health Insurance Data Service website (http:/ / nhiss.nhis.or.kr). The raw data cannot be retrieved from the server unless the researchers submit a study proposal for acquiring approval from each institutional review board, which is also reviewed by the NHIS review committee to access to the database.

Acknowledgments: Data use was approved by Korea National Health Insurance, and the results do not necessarily represent the opinion of the National Health Insurance Corporation.

Conflicts of Interest: The authors declare no conflict of interest. 


\section{References}

1. Saeedi, P.; Petersohn, I.; Salpea, P.; Malanda, B.; Karuranga, S.; Unwin, N.; Colagiuri, S.; Guariguata, L.; Motala, A.A.; Ogurtsova, K.; et al. Global and regional diabetes prevalence estimates for 2019 and projections for 2030 and 2045: Results from the International Diabetes Federation Diabetes Atlas, 9(th) edition. Diabetes Res. Clin. Pr. 2019, 157, 107843. [CrossRef] [PubMed]

2. Maggio, C.A.; Pi-Sunyer, F.X. Obesity and type 2 diabetes. Endocrinol. Metab. Clin. North Am. 2003, 32, 805-822. [CrossRef]

3. Kahn, S.E.; Hull, R.L.; Utzschneider, K.M. Mechanisms linking obesity to insulin resistance and type 2 diabetes. Nature 2006, 444, 840-846. [CrossRef]

4. Haslam, D.W.; James, W.P. Obesity. Lancet 2005, 366, 1197-1209. [CrossRef]

5. Jensen, M.D.; Ryan, D.H.; Apovian, C.M.; Ard, J.D.; Comuzzie, A.G.; Donato, K.A.; Hu, F.B.; Hubbard, V.S.; Jakicic, J.M.; Kushner, R.F.; et al. 2013 AHA/ACC/TOS guideline for the management of overweight and obesity in adults: A report of the American College of Cardiology / American Heart Association Task Force on Practice Guidelines and The Obesity Society. Circulation 2014, 129, S102-S138. [CrossRef] [PubMed]

6. American Diabetes Association. Standards of Medical Care in Diabetes-2016 Abridged for Primary Care Providers. Clin. Diabetes 2016, 34, 3-21. [CrossRef] [PubMed]

7. Sternfeld, B.; Wang, H.; Quesenberry, C.P., Jr.; Abrams, B.; Everson-Rose, S.A.; Greendale, G.A.; Matthews, K.A.; Torrens, J.I.; Sowers, M. Physical activity and changes in weight and waist circumference in midlife women: Findings from the Study of Women's Health Across the Nation. Am. J. Epidemiol. 2004, 160, 912-922. [CrossRef] [PubMed]

8. Karvonen-Gutierrez, C.; Kim, C. Association of Mid-Life Changes in Body Size, Body Composition and Obesity Status with the Menopausal Transition. Healthcare 2016, 4, 42. [CrossRef]

9. Kautzky-Willer, A.; Harreiter, J.; Pacini, G. Sex and Gender Differences in Risk, Pathophysiology and Complications of Type 2 Diabetes Mellitus. Endocr. Rev. 2016, 37, 278-316. [CrossRef]

10. Slopien, R.; Wender-Ozegowska, E.; Rogowicz-Frontczak, A.; Meczekalski, B.; Zozulinska-Ziolkiewicz, D.; Jaremek, J.D.; Cano, A.; Chedraui, P.; Goulis, D.G.; Lopes, P.; et al. Menopause and diabetes: EMAS clinical guide. Maturitas 2018, 117, 6-10. [CrossRef]

11. Park, S.K.; Harlow, S.D.; Zheng, H.; Karvonen-Gutierrez, C.; Thurston, R.C.; Ruppert, K.; Janssen, I.; Randolph, J.F., Jr. Association between changes in oestradiol and follicle-stimulating hormone levels during the menopausal transition and risk of diabetes. Diabet. Med. 2017, 34, 531-538. [CrossRef] [PubMed]

12. Brand, J.S.; van der Schouw, Y.T.; Onland-Moret, N.C.; Sharp, S.J.; Ong, K.K.; Khaw, K.T.; Ardanaz, E.; Amiano, P.; Boeing, H.; Chirlaque, M.D.; et al. Age at menopause, reproductive life span, and type 2 diabetes risk: Results from the EPIC-InterAct study. Diabetes Care 2013, 36, 1012-1019. [CrossRef] [PubMed]

13. Pu, D.; Tan, R.; Yu, Q.; Wu, J. Metabolic syndrome in menopause and associated factors: A meta-analysis. Climacteric 2017, 20, 583-591. [CrossRef] [PubMed]

14. Randolph, J.F., Jr.; Zheng, H.; Sowers, M.R.; Crandall, C.; Crawford, S.; Gold, E.B.; Vuga, M. Change in follicle-stimulating hormone and estradiol across the menopausal transition: Effect of age at the final menstrual period. J. Clin. Endocrinol. Metab. 2011, 96, 746-754. [CrossRef] [PubMed]

15. Lee, D.H.; Keum, N.; Hu, F.B.; Orav, E.J.; Rimm, E.B.; Willett, W.C.; Giovannucci, E.L. Comparison of the association of predicted fat mass, body mass index, and other obesity indicators with type 2 diabetes risk: Two large prospective studies in US men and women. Eur. J. Epidemiol. 2018, 33, 1113-1123. [CrossRef] [PubMed]

16. Cheol Seong, S.; Kim, Y.Y.; Khang, Y.H.; Heon Park, J.; Kang, H.J.; Lee, H.; Do, C.H.; Song, J.S.; Hyon Bang, J.; Ha, S.; et al. Data Resource Profile: The National Health Information Database of the National Health Insurance Service in South Korea. Int. J. Epidemiol. 2017, 46, 799-800. [CrossRef]

17. Pan, W.H.; Yeh, W.T. How to define obesity? Evidence-based multiple action points for public awareness, screening, and treatment: An extension of Asian-Pacific recommendations. Asia Pac. J. Clin. Nutr. 2008, 17, 370-374. [PubMed]

18. WHO. Obesity: Preventing and managing the global epidemic. Report of a WHO consultation. World Health Organ. Tech. Rep. Ser. 2000, 894, 1-253.

19. Kapoor, E.; Collazo-Clavell, M.L.; Faubion, S.S. Weight Gain in Women at Midlife: A Concise Review of the Pathophysiology and Strategies for Management. Mayo Clin. Proc. 2017, 92, 1552-1558. [CrossRef] [PubMed]

20. Cifkova, R.; Pitha, J.; Krajcoviechova, A.; Kralikova, E. Is the impact of conventional risk factors the same in men and women? Plea for a more gender-specific approach. Int. J. Cardiol. 2019, 286, 214-219. [CrossRef]

21. De Pergola, G.; Maldera, S.; Tartagni, M.; Pannacciulli, N.; Loverro, G.; Giorgino, R. Inhibitory effect of obesity on gonadotropin, estradiol, and inhibin B levels in fertile women. Obes. Silver Spring 2006, 14, 1954-1960. [CrossRef] [PubMed]

22. Freeman, E.W.; Sammel, M.D.; Lin, H.; Gracia, C.R. Obesity and reproductive hormone levels in the transition to menopause. Menopause 2010, 17, 718-726. [CrossRef]

23. Nelson, L.R.; Bulun, S.E. Estrogen production and action. J. Am. Acad. Derm. 2001, 45, S116-S124. [CrossRef] [PubMed]

24. Greendale, G.A.; Sternfeld, B.; Huang, M.; Han, W.; Karvonen-Gutierrez, C.; Ruppert, K.; Cauley, J.A.; Finkelstein, J.S.; Jiang, S.F.; Karlamangla, A.S. Changes in body composition and weight during the menopause transition. JCI Insight 2019, 4(5), e124865. [CrossRef]

25. Tatsumi, Y.; Ohno, Y.; Morimoto, A.; Nishigaki, Y.; Mizuno, S.; Watanabe, S. Age differences in the risk of diabetes incidence according to body mass index level in Japanese women. Obes. Res. Clin. Pr. 2013, 7, e455-e463. [CrossRef] [PubMed] 
26. Asia Pacific Cohort Studies Collaboration; Ni Mhurchu, C.; Parag, V.; Nakamura, M.; Patel, A.; Rodgers, A.; Lam, T.H. Body mass index and risk of diabetes mellitus in the Asia-Pacific region. Asia Pac. J. Clin. Nutr. 2006, 15, 127-133. [PubMed]

27. Manrique-Acevedo, C.; Chinnakotla, B.; Padilla, J.; Martinez-Lemus, L.A.; Gozal, D. Obesity and cardiovascular disease in women. Int. J. Obes. Lond. 2020, 44, 1210-1226. [CrossRef] [PubMed]

28. Manson, J.E.; Rimm, E.B.; Colditz, G.A.; Willett, W.C.; Nathan, D.M.; Arky, R.A.; Rosner, B.; Hennekens, C.H.; Speizer, F.E.; Stampfer, M.J. A prospective study of postmenopausal estrogen therapy and subsequent incidence of non-insulin-dependent diabetes mellitus. Ann. Epidemiol. 1992, 2, 665-673. [CrossRef]

29. Margolis, K.L.; Bonds, D.E.; Rodabough, R.J.; Tinker, L.; Phillips, L.S.; Allen, C.; Bassford, T.; Burke, G.; Torrens, J.; Howard, B.V.; et al. Effect of oestrogen plus progestin on the incidence of diabetes in postmenopausal women: Results from the Women's Health Initiative Hormone Trial. Diabetologia 2004, 47, 1175-1187. [CrossRef] [PubMed]

30. Kanaya, A.M.; Herrington, D.; Vittinghoff, E.; Lin, F.; Grady, D.; Bittner, V.; Cauley, J.A.; Barrett-Connor, E. Glycemic effects of postmenopausal hormone therapy: The Heart and Estrogen/progestin Replacement Study. A randomized, double-blind, placebo-controlled trial. Ann. Intern. Med. 2003, 138, 1-9. [CrossRef] [PubMed]

31. Flegal, K.M.; Shepherd, J.A.; Looker, A.C.; Graubard, B.I.; Borrud, L.G.; Ogden, C.L.; Harris, T.B.; Everhart, J.E.; Schenker, N. Comparisons of percentage body fat, body mass index, waist circumference, and waist-stature ratio in adults. Am. J. Clin. Nutr. 2009, 89, 500-508. [CrossRef] [PubMed] 